\title{
A NEWLY FORMING COLD FLOW PROTOGALACTIC DISK, A SIGNATURE OF COLD ACCRETION FROM THE COSMIC WEB
}

\author{
D. Christopher Martin ${ }^{1}$, Mateusz Matuszewski ${ }^{1}$, Patrick Morrissey ${ }^{1}$, James D. Neill $^{1}$, \\ Anna Moore ${ }^{2}$, Charles C. Steidel ${ }^{3}$, and Ryan Trainor ${ }^{4}$ \\ ${ }^{1}$ Cahill Center for Astrophysics, California Institute of Technology, 1216 East California Boulevard, \\ Mail Code 278-17, Pasadena, California 91125, USA; cmartin@srl.caltech.edu. \\ ${ }^{2}$ Caltech Optical Observatories, Cahill Center for Astrophysics, California Institute of Technology, 1216 East California Boulevard, \\ Mail Code 11-17, Pasadena, California 91125, USA \\ ${ }^{3}$ Cahill Center for Astrophysics, California Institute of Technology, 1216 East California Boulevard, Mail Code 249-17, Pasadena, California 91125, USA \\ ${ }^{4}$ Department of Astronomy, University of California, Berkeley, 50115 Campbell Hall, Berkeley, CA 94720, USA \\ Received 2016 February 23; revised 2016 April 11; accepted 2016 April 13; published 2016 June 3
}

\begin{abstract}
How galaxies form from, and are fueled by, gas from the intergalactic medium (IGM) remains one of the major unsolved problems in galaxy formation. While the classical Cold Dark Matter paradigm posits galaxies forming from cooling virialized gas, recent theory and numerical simulations have highlighted the importance of cold accretion flows - relatively cool $(T \sim$ few $\times 104 \mathrm{~K})$ unshocked gas streaming along filaments into dark matter halos, including hot, massive, high-redshift halos. These flows are thought to deposit gas and angular momentum into the circumgalactic medium resulting in disk- or ring-like structures, eventually coalescing into galaxies forming at filamentary intersections. We earlier reported a bright, Ly $\alpha$ emitting filament near the QSO HS1549+19 at redshift $z=2.843$ discovered with the Palomar Cosmic Web Imager. We now report that the bright part of this filament is an enormous $(R>100 \mathrm{kpc})$ rotating structure of hydrogen gas with a disk-like velocity profile consistent with a $4 \times 10^{12} M_{\odot}$ halo. The orbital time of the outer part of the what we term a "protodisk" is comparable to the virialization time and the age of the universe at this redshift. We propose that this protodisk can only have recently formed from cold gas flowing directly from the cosmic web.
\end{abstract}

Key words: dark matter - galaxies: evolution - galaxies: formation - galaxies: high-redshift - intergalactic medium - quasars: general

\section{INTRODUCTION}

In the standard model of galaxy formation in the Cold Dark Matter paradigm, collapsing, virialized gas forms a hot halo and slowly cools to fuel star and galaxy formation at the halo center (Blumenthal et al. 1984). This hot accretion model was challenged by the discovery of a population of rapidly star forming galaxies at early times (Steidel et al. 1996) and in the last 12 years by the "cold accretion model" (Birnboim \& Dekel 2003; Kereš et al. 2005; Dekel \& Birnboim 2006; Dekel et al. 2009) motivated by theory and numerical simulations. Cold accretion flows transport $\sim 10^{4} \mathrm{~K}$ gas from cosmic web filaments to galactic disks, offering a direct and high-mass flux channel for delivering fuel for star formation. Recent simulations (Pichon et al. 2011; Powell et al. 2011; Stewart et al. 2011, 2013; Tillson et al. 2012; Codis et al. 2013; Danovich et al. 2015) predict that inflowing gas and dark matter will be subject to strong tidal torques outside the halo. The narrow, cold streams of inflowing gas have higher quadropole moment than the more extended dark matter, respond more strongly to the torques, and therefore enter the halo virial radius with substantial angular momentum exceeding that of the inflowing dark matter. The spinning gas sinks to the central protogalaxy in an orbital time (Danovich et al. 2015). The result is an extended, "cold-flow disk" or "cold spiral inflows" with substantial angular momentum and column density. The angular momentum alignment between inflowing gas and the central disk is weak at the virial radius but increases as the gas approaches the disk (Danovich et al. 2012, 2015).

We recently reported the discovery of just such a "cold-flow disk" illuminated by the QSO UM287 (Martin et al. 2015, hereafter M15). This discovery follows on growing evidence for rotating systems in Ly $\alpha$ nebula (Prescott et al. 2012, 2015). In this Letter, we report a reanalysis of Palomar Cosmic Web Imager (PCWI) data on QSO HS1549+1919 first presented by Martin et al. (2014a). The data show that the brightest part of the HS1549+1919 filament is consistent with a second giant gaseous rotating structure, with substantial angular momentum, illuminated by the nearby QSO, and fed by filaments. We will show that this object shows properties like "cold-flow disks" and "cold spiral inflows" (Pichon et al. 2011; Stewart et al. 2011, 2013; Danovich et al. 2012, 2015). While a number of possible names could be imagined, for the purpose of this discussion of our empirical results, we label such an object a "cold-flow protogalactic disk" or "protodisk" for short.

\section{OBSERVATIONS}

We performed the observations of QSO HS1549+1919 $(z=2.843)$ using the PCWI, an instrument designed to search for, map, and characterize IGM emission and other low surface brightness phenomena (Matuszewski et al. 2010). We observed the region around the QSO that is known to harbor extensive narrowband Ly $\alpha$ emission (Trainor \& Steidel 2012). We obtained a total of $6.5 \mathrm{hr}$ on-source and $6.5 \mathrm{hr}$ off-source exposure centered on QSO HS1549+19 $\left(15^{\mathrm{h}} 51^{\mathrm{m}} 52.48^{\mathrm{s}}\right.$, $+19^{\circ} .11^{\prime} 4$ !' 2 ), over a total region 120 arcsec east-west by 60 arcsec north-south.

The methodology and details of our observations and data analysis are described extensively elsewhere (Martin et al. 2014a, hereafter M14a; Martin et al. 2014b, hereafter M14b). The endproduct of the data reduction of the co-added PCWI 


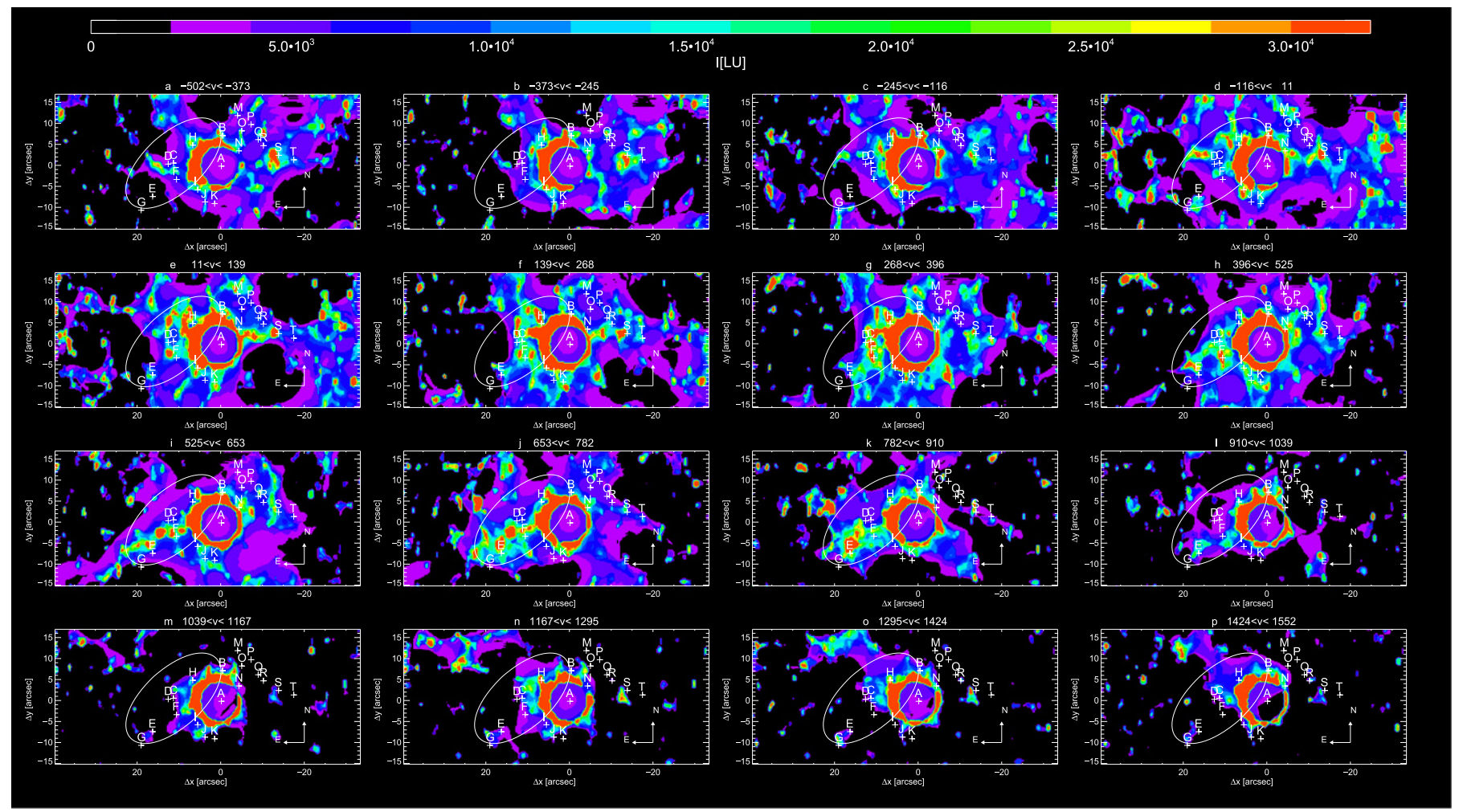

Figure 1. Channel maps of the HS1549+1919 data cube. Panels show channel maps in $2 \AA\left(170 \mathrm{~km} \mathrm{~s}^{-1}\right)$ bins from $v=\left(-502\right.$ to $\left.-373 \mathrm{~km} \mathrm{~s}{ }^{-1}\right)$ to $v=(+1424$ to $+1553 \mathrm{~km} \mathrm{~s}^{-1}$ ). Intensity scale is given by color bar. Sources in the field are shown by letters and crosses. The QSO has been masked. A white ellipse shows the bestfit location of the disk. The disk starts in panel (d), upper right of the ellipse and ends in the lower left in panels (k) and (1). Intensity units are LU with 10,000 $\mathrm{LU}=1 \times 10^{-18} \mathrm{erg} \mathrm{cm}^{-2} \mathrm{~s}^{-1} \operatorname{arcsec}^{-2}$.

observations is an adaptively smoothed flux cube (R.A., decl., $\lambda$ ). We showed in Paper I (M14a) that there is a bright extended filament detected in the data (M14a, Figure 21). The filament is fainter far from the QSO and shows a narrow kinematic width. There is also evidence in this figure for a significant velocity shear in the emission nearing the QSO. After discovering a clear giant protogalactic disk in the UM287 nebula (M15), we returned to this object and noted that the strong velocity shear is consistent with a large rotating disk. In particular, channel cuts through the smoothed data cube (Figure 1) illustrate a disk morphology (in the sense that successive redshifted slices show emission moving from one side of a disk to the other). In M14a, we demonstrated that nearby line and continuum objects are not responsible for the emission near the QSO. Using the same considerations as discussed in M15, we have verified that artifacts of QSO subtraction do not explain the disk-like emission.

A series of narrowband and velocity images, pseudo-slit spectra, and extracted 1D kinematics is shown in Figure 2. Figure 2(a) shows a narrowband image offset from the systemic redshift by $+360 \mathrm{~km} \mathrm{~s}^{-1}$ with a bandwidth of $14 \AA( \pm 400$ $\mathrm{km} \mathrm{s}^{-1}$ ). Figure 2(b) shows the mean velocity within this band. We note that the region of brightest emission, outlined in Figure 2(a), is also the region of maximum velocity shear, shown in Figure 2(b). We show a pseudo-slit in Figure 2(c) designed to traverse this region of bright emission and shear, from which we extract the spectral image of Figure 2(d). The spectral image shows a clear disk-like rotation curve. We define a sheared velocity window based on this rotation curve illustrated in Figure 2(d) and used to generate the narrowband image of Figure 2(c) and the velocity map of Figure 2(b).
Within this sheared window, we extract the mean velocity and velocity dispersion profile in Figure 2(e).

\section{ANALYSIS}

The narrowband and spectral images reveal an extended nebula offset from and illuminated by the nearby QSO (Figure 3(a)) that is consistent with a rotating structure that may be an incomplete, forming disk and associated inflowing streams ("cold-flow protodisk" or "protodisk" for short). In order to secure this identification, we first fit the nebula with a combined 2D velocity profile appropriate for a disk and surface brightness model. The velocity profile is fit using an NFW dark matter halo, while the surface brightness distribution is modeled with a smooth exponential disk, and a central deficit. The central deficit only impacts the intensity fit and is not used to exclude pixels for the velocity fit. The disk position and velocity center, the inclination, position angle, halo mass, halo concentration, disk scale length, and hole radius are all free parameters in the fit. The model includes the effects of slit averaging and the instrument and seeing point-spread function measured using the QSO. We use an intensity cut to exclude low signal-to-noise date. We find similar results when excluding all pixels with lower than $10 \%, 20 \%$, or $30 \%$ of the maximum intensity. This corresponds to areas of 550, 330, or $220 \operatorname{arcsec}^{2}$. Results are quoted for a $10 \%$ cut.

We find that the nebula has a $2 \mathrm{D}$ velocity profile well fit by a rotating disk in a dark matter halo with mass $\log _{10}$ $M_{h}=12.6 \pm 0.2$, concentration $c=4.4 \pm 2.6$, circular velocity (at the virial radius) $\sim 350 \mathrm{~km} \mathrm{~s}^{-1}$, and virial radius $125 \mathrm{kpc}$. The nebula has a major axis diameter in the range of 


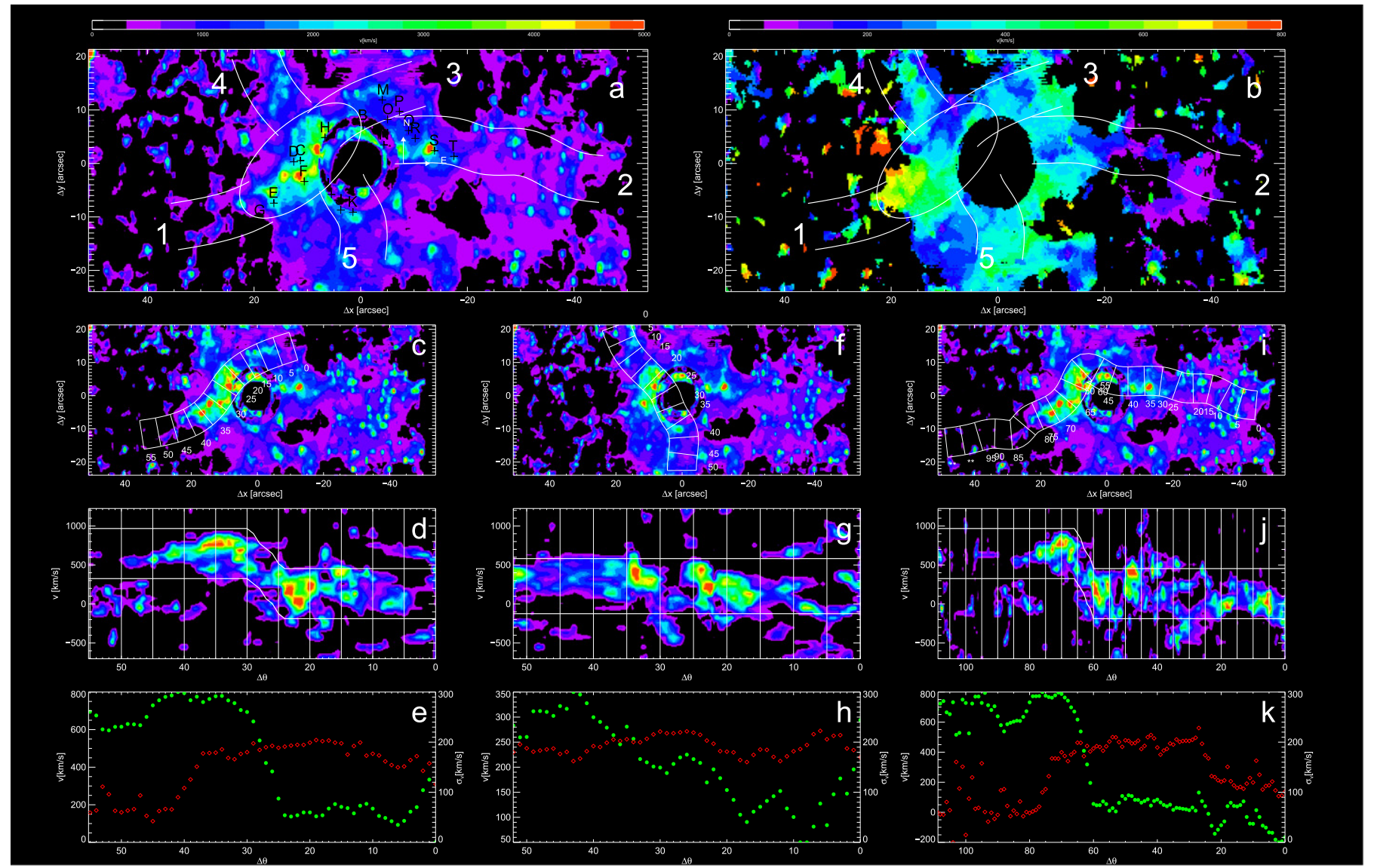

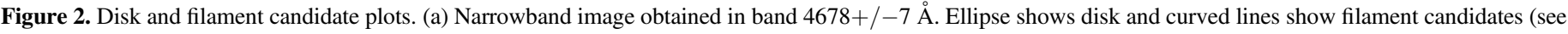

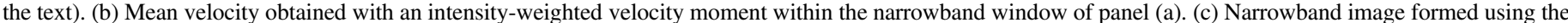

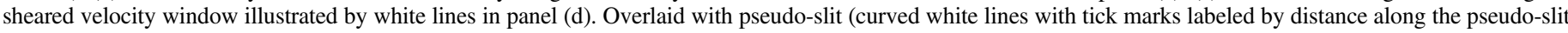

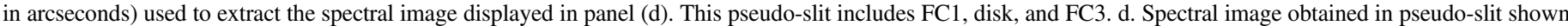

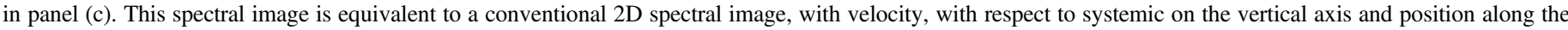

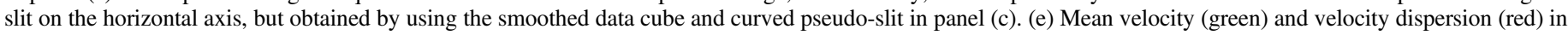

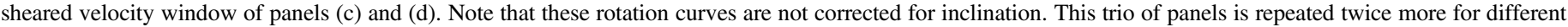

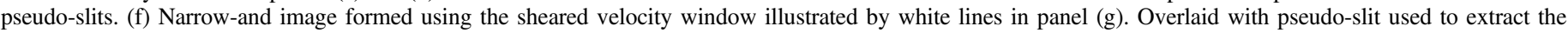

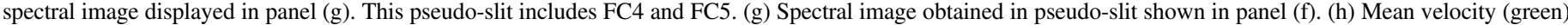

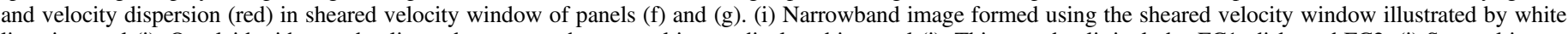

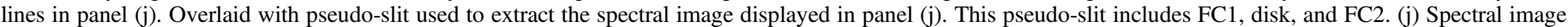
obtained in pseudo-slit shown in panel (i). (k) Mean velocity (green) and velocity dispersion (red) in sheared velocity window of panels (i) and (j).

20-30 arcsec or 160-230 kpc. Thus, the nebula extends nearly to the virial radius. There is a central deficit of radius of $20 \pm$ $10 \mathrm{kpc}$ and essentially infinite scale length (flat radial profile). This flat profile suggests that the nebula is not a classical exponential disk. It is inclined by $\sim 60^{\circ}$ to the plane of the sky. Our Figure 3 is a series of zoom panels showing the data and the 2D model fits and residuals. We also show velocity linewidths over the object.

Because rotational motion may be accompanied by radial flows in an inspiraling structure, we investigated the impact of adding a radial flow to the $2 \mathrm{D}$ model. Typically, we find that a flow of $80-100 \mathrm{~km} \mathrm{~s}^{-1}$ improves the fit moderately (reducing the total $\chi^{2}$ by anywhere from 1 to 5 with this single additional parameter) without changing the halo mass or concentration significantly. For a full disk, there is ambiguity as to the sign of the radial flow (inflow versus outflow) depending on whether apparent circulation is clockwise or counterclockwise. This ambiguity is removed if the nebula includes a forming disk and an inspiraling filament. If the southeast extension of the nebula (filament candidate (FC) 1 (see below) and the southeast portion of the bright nebula (cf. Figures 5(b) and (c) and discussion below)) is this inspiraling flow, then the apparent direction of circulation is counterclockwise and the radial flow is an inflow.

Using the pseudo-slit (Figure 2(c)), we extract a 1D average velocity profile (Figures 2(e), 4(a)) that is well fit by the same 2D model (without radial flow) with consistent inferred mass $\log _{10} M_{h}=12.5 \pm 0.1$ and concentration $c=7.6 \pm 2.5$. The disk rotation curve is well fit for $R<120 \mathrm{kpc}$, beyond which the velocity falls. The velocity dispersion $\sigma_{v}$ (Figure 4(b)) also falls from $180 \mathrm{~km} \mathrm{~s}^{-1}$ to about $60 \mathrm{~km} \mathrm{~s}^{-1}$. We return to this feature below.

We note that the derived dark halo mass agrees with the median mass, $\log \left(M_{h}\right)=12.3 \pm 0.5$, derived for hyperluminous QSOs (including QSO1549+19, among the most luminous) from clustering by Trainor \& Steidel (2012). While we did not model the protodisk as centered on the QSO, and the QSO is neither spatially nor kinematically centered on the protodisk, the protodisk and the QSO may share the same 


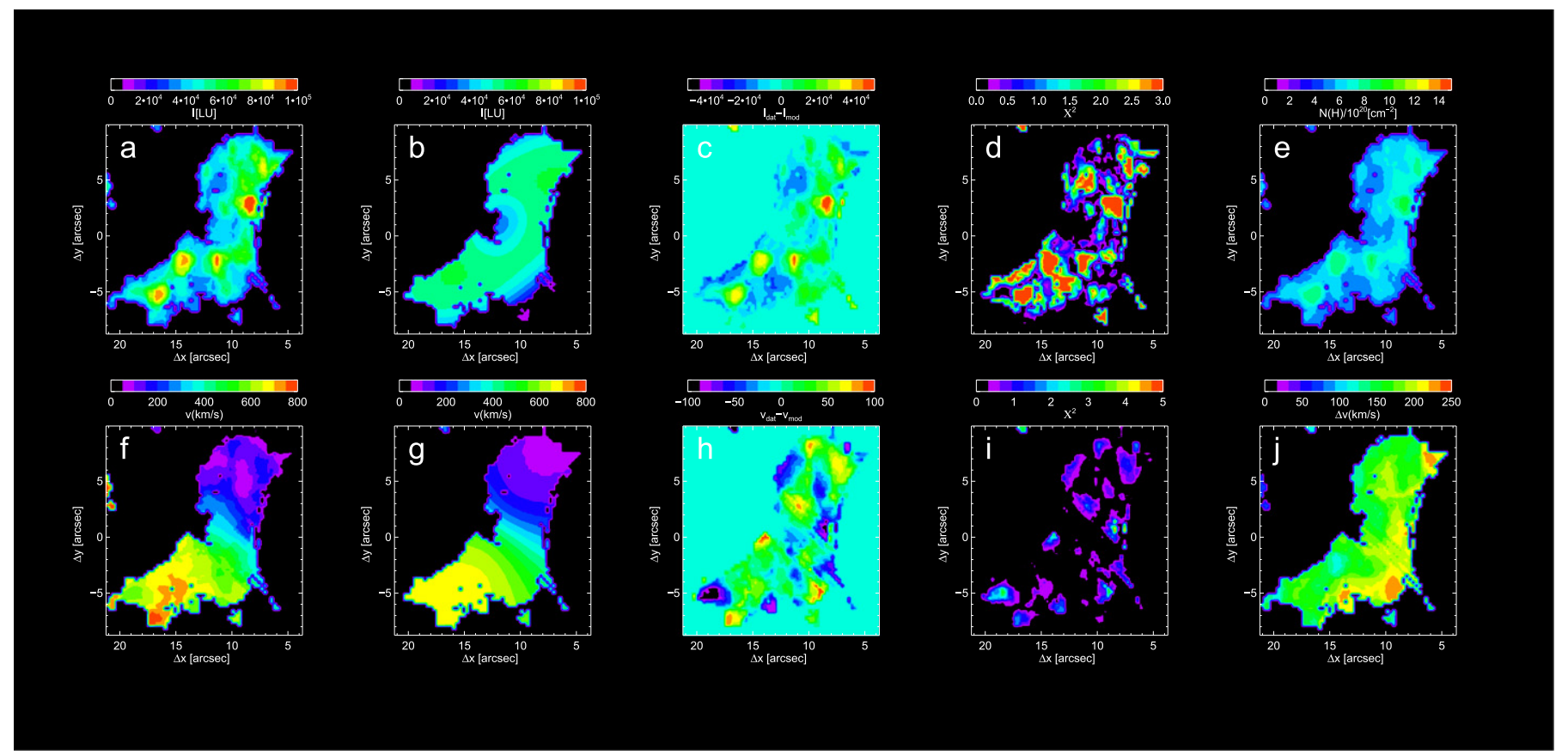

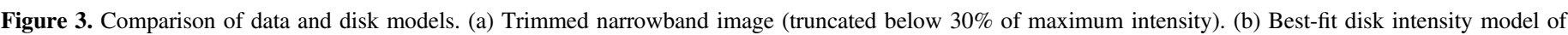

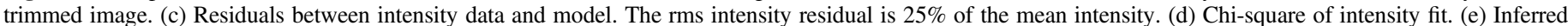

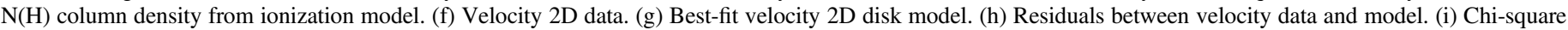
of velocity fit. (j) Velocity dispersion map.

(possibly composite) halo or be hosted by two nearby halos destined to merge.

The protodisk is assumed to be illuminated by the nearby QSO, based on arguments presented in M15. There is almost no dependence on QSO/disk distance, which must be at least of the order of the disk diameter or $\sim 200 \mathrm{kpc}$. We used Cloudy (Ferland et al. 2013) to model the emission and infer gas physical parameters. The result is the Lyman continuum optical depth is $\ll 1$, the ionization fraction is high, and the flux is only a function of thickness and column density (or thickness and intrinsic density; Bertoldi 1989; Hennawi \& Prochaska 2013). If significant dust $\left(>10^{-3}-10^{-2}\right.$ of the standard Milky Way ratio) were present, the fluorescent $\operatorname{Ly} \alpha$ would be much dimmer (Neufeld 1990). This suggests a low dust-to-gas ratio and metallicity in the nebula. Taking a canonical disk thickness of $t_{d}=3 \mathrm{kpc}$, the column density is $\log \left(N_{H}\right)=20.75+0.5 \log$ $(I / 60,000 L U)+0.5 \log \left(t_{d} / 3 \mathrm{pkpc}\right.$ ) (see M15 for derivation and discussion). The column density distribution is given in Figures 3(e) and 4(c), and the cold baryonic and dark matter mass distributions are compared in Figures 4(d) and (e). The cold baryonic mass $\left(M_{b \text {, disk }} \sim 10^{11} M_{\odot}\right)$ is about $20 \%$ of the universal baryon fraction of 0.17 , similar to the stellar mass to dark matter fraction (Behroozi et al. 2010), suggesting that the cold inflowing gas may eventually form stars with high efficiency.

We can estimate the total angular momentum, specific angular momentum, and normalized spin parameter using the kinematics and estimated column density. These are $L_{b}=1.8 \times 10^{15}\left(t_{d} / 3 \mathrm{kpc}\right)^{0.5} \quad M_{\odot} \mathrm{kpc} \mathrm{km} \mathrm{s}^{-1}, j_{b}=L_{b} / M_{b}=$ $1.8 \times 10^{4} \quad \mathrm{kpc} \mathrm{km} \mathrm{s}{ }^{-1}$, and $\lambda_{b}=j_{b} /\left(\sqrt{2} R_{\mathrm{vir}} v_{\mathrm{vir}}\right)=0.30$, respectively. Note that the cold baryon mass, and therefore the angular momentum, scales as the square root of the thickness. Simulated dark matter halos have spin parameters of with little redshift or mass dependence, and with a log-normal distribution that can reach $\lambda_{d} \sim 0.1$ at $2 \sigma$ above the mean (Bullock et al. 2001; Stewart et al. 2013; Danovich et al. 2015). We estimate a baryonic spin parameter that is three times higher, indicating that the baryons in the nebula have specific angular momentum substantially higher than the dark matter halo.

Our data indicate we may have detected filaments feeding the protodisk. Returning to Figure 2, we see in panels (a) and (b) that there are several extensions that could be filaments connecting to the disk. Alternatively, they could be outflows either from the disk or the QSO, or gas in orbit in the system. We indicate in Figures 2(a) and (b) five "FCs" labeled with large numbers and try to determine properties that may be indicative of filamentary inflow to the disk. The key point that is demonstrated in Figure 2 is that, as in the case of UM287 (M15) and LAB2 (M14b), the extended FCs share mean velocities with that of the central object at the location where the filament meets the central object.

It is plausible that an inflowing filament would show a sudden increase in velocity dispersion at decreasing radii (and unlikely that an outflow would show a decrease at increasing radius). Reasons include the superposition of filament inflow and disk kinematics (Ceverino et al. 2010), shocks, and mixing at the filament/disk interface, or the cold stream/hot CGM interface. Radial kinetic energy must be dissipated in order to produce circularized motion. FC1 extends east from gridline 40-50 arcsec in Figures 2(c)-(e). It appears faint, perhaps due to only partial illumination by the QSO. From Figure 2(e), we see that the velocity dispersion changes from $\sim 50 \mathrm{~km} \mathrm{~s}^{-1}$ to $200 \mathrm{~km} \mathrm{~s}^{-1}$ moving inward, and the mean velocity from 780 $\mathrm{km} \mathrm{s}^{-1}$ (at the disk edge) to $600 \mathrm{~km} \mathrm{~s}^{-1}$. This could be due to superposition of multiple velocity components or a real increase in turbulent velocity in the inflowing gas. FC2 appears in Figures 2(i)-(k) over gridlines $0-40$ arcsec and shows a 


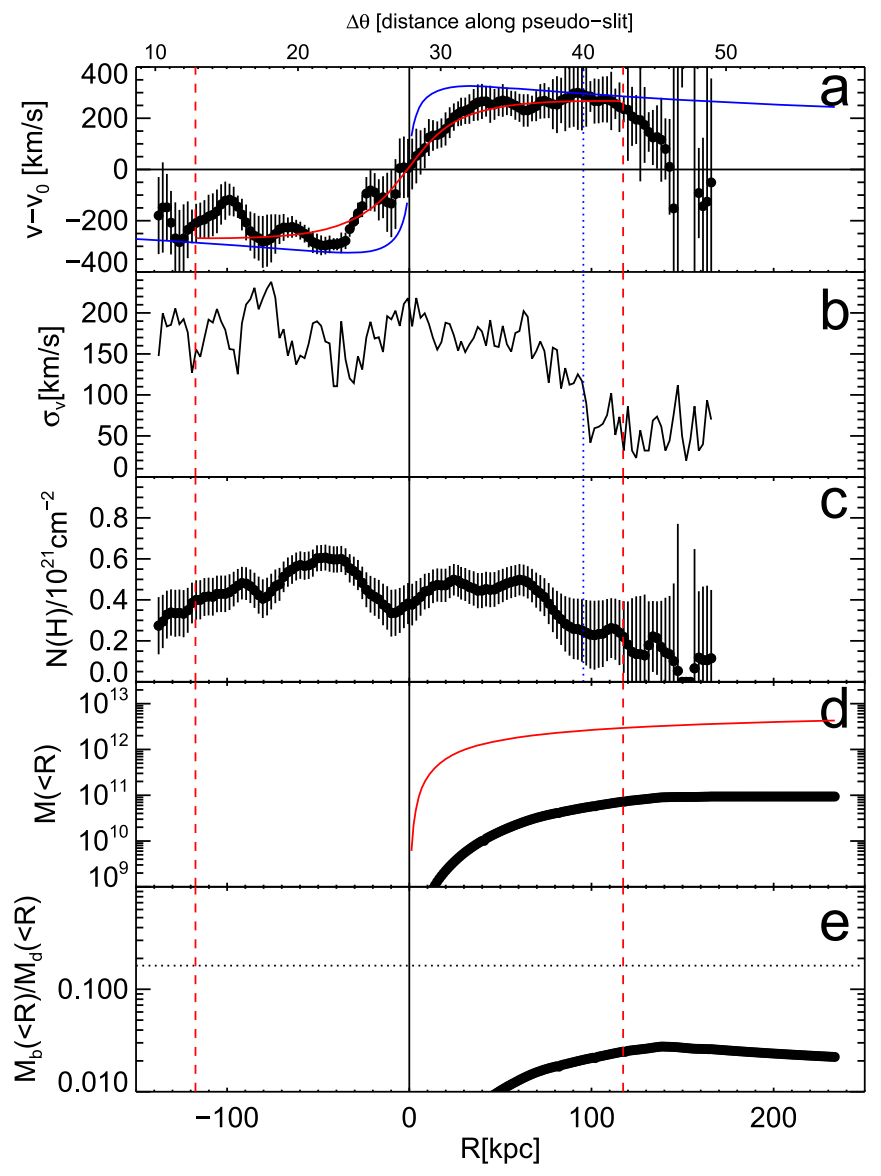

Figure 4. Physical properties of extended disk. (a) Mean velocity along the pseudo-slit (Figure 2), with vertical dashed lines showing $\sim 125 \mathrm{kpc}$ extent of disk (dotted) and virial radius (red). Filament profile is $80<R[\mathrm{kpc}]<250$. NFW profile fit (red line includes PSF convolution, blue without PSF). Error bars are $\pm 1 \sigma$. (b) Velocity dispersion in pseudo-slit/sheared velocity window. (c) Inferred hydrogen column densities assuming a $3 \mathrm{kpc}$ disk thickness. Error bars do not include uncertainty due to disk thickness. (d) Baryonic (dots) and dark matter (red line) mass inside radius $R$. (e) Ratio of baryonic mass inside $\mathrm{R}$ to dark matter mass compared to canonical baryon to dark matter mass ratio of 0.17 (horizontal dotted line).

distinct drop in velocity dispersion at $25 \operatorname{arcsec}$ to $\sim 100$ $\mathrm{km} \mathrm{s}^{-1}$. These filament definitions were used in M14a, although as we have shown, the brightest part of filament 1 appears as part of a protodisk. The other three FCs (3, 4, and 5) show a velocity dispersion $\sim 150-200 \mathrm{~km} \mathrm{~s}^{-1}$, comparable to that in the disk. FC4 and FC5 show velocity gradients in the same orientation as the nebula, and thus could be contributing additional angular momentum. The change in FC2 is more distant from the likely boundary, although the interface zone is obscured by the QSO. FC2 connects to the bright line and continuum source $\mathrm{S}$ and may connect directly to the QSO. Thus, the case for FC2 feeding the disk is not as clear as FC1.

We have used a notional inflow model to investigate the filament/disk geometry and kinematics. It is illustrative and not meant to be exhaustive. Coplanar filaments of gas inflow into an NFW halo, with an finite impact parameter and angular momentum, leading to disk formation. Radial velocity is damped with a radial drag acceleration $a_{r}=b_{r} v_{r}$ with $b_{r}=(100 \mathrm{Myr})^{-1}$. In Figure 5, we show kinematic maps for three cases using this model: (1) a disk formed by two filaments, (2) a disk just forming from one filament, and (3) a partially formed disk from one filament. These models show features consistent with the observations, including the velocity step at the filament/disk transition (due to the difference between the line of sight projected velocity of the inflowing filament and the returning, rotating disk material) and the increase in velocity dispersion (due both to superposition of gas in filament and disk and possible shocks at the interface not included in the model). The incomplete disk cases (Figures 5(b) and (c)) give a possible explanation for the lower intensity northeast sector of the observed disk. FC4 and FC5 are not easily reproduced by co-planar filaments (and in simulations filaments are not necessarily co-planar; e.g., Danovich et al. 2012), and we defer a more complex model to an analysis of deeper, higher-resolution data we will obtain from KCWI.

\section{COLD FLOW PROTOGALACTIC DISK CRITERIA}

Alternative models for the Ly $\alpha$ nebula can be offered. The tidal tail explanation was discussed in M15 and shown to be less likely, and the same considerations apply here. Another model is a pure inflowing filament without significant rotation or disk formation. In this case, we might expect a velocity gradient due to accelerated infall, modified by any drag that might be present due to interaction with virialized hot halo gas. Without drag, accelerated infall would produce a significant velocity curvature (versus a constant velocity derivative) as the filament approaches the halo center. Also, for a constant filament density the gas density and emission intensity is more likely to decrease as the flow velocity increases. We would not expect a density/intensity concentration at the velocity center, and we would not expect abrupt discontinuities in the velocity profile as we see at the disk edge. Significantly, cold inflow simulations show gas inflow that does not accelerate (consistent with our observations) and instead releases gravitational potential energy in radiation (dominated by Ly $\alpha$; Goerdt et al. 2010).

We can codify these expectations by enumerating a set of protodisk criteria based on considerations discussed here and in M15. We posit that objects exhibiting a large fraction of these features are more likely to be cold-flow protogalactic disks.

1. Higher intensity symmetrically located around 1D velocity center and relatively uniform intensity with clear intensity break at edges;

2. Near constant slope velocity gradient (1D) consistent with an NFW halo;

3. 2D velocity and intensity distribution consistent with a disk in an NFW halo with minimal residuals;

4. Evidence for one or more filaments with slow velocity gradient and possibly lower-velocity dispersion;

5. Abrupt kinematic transition at disk edge (versus continuous acceleration expected from single-filament dragfree infall) either in mean velocity or velocity dispersion or both consistent with filament/disk interface

6. Star formation at center of disk co-located with a possible intensity/gas deficit; and

7. Kinematics consistent with radial and spiral inflow.

In the case of QSO1549+19, all of these criteria are present with the following provisos. For criterion 3, as discussed above, the 2D intensity distribution is not centered symmetrically on the $2 \mathrm{D}$ velocity center, consistent with an empty or unilluminated sector of a possibly partial disk. This was also the case for the UM287 disk (M15). Also, for criterion 6, we 


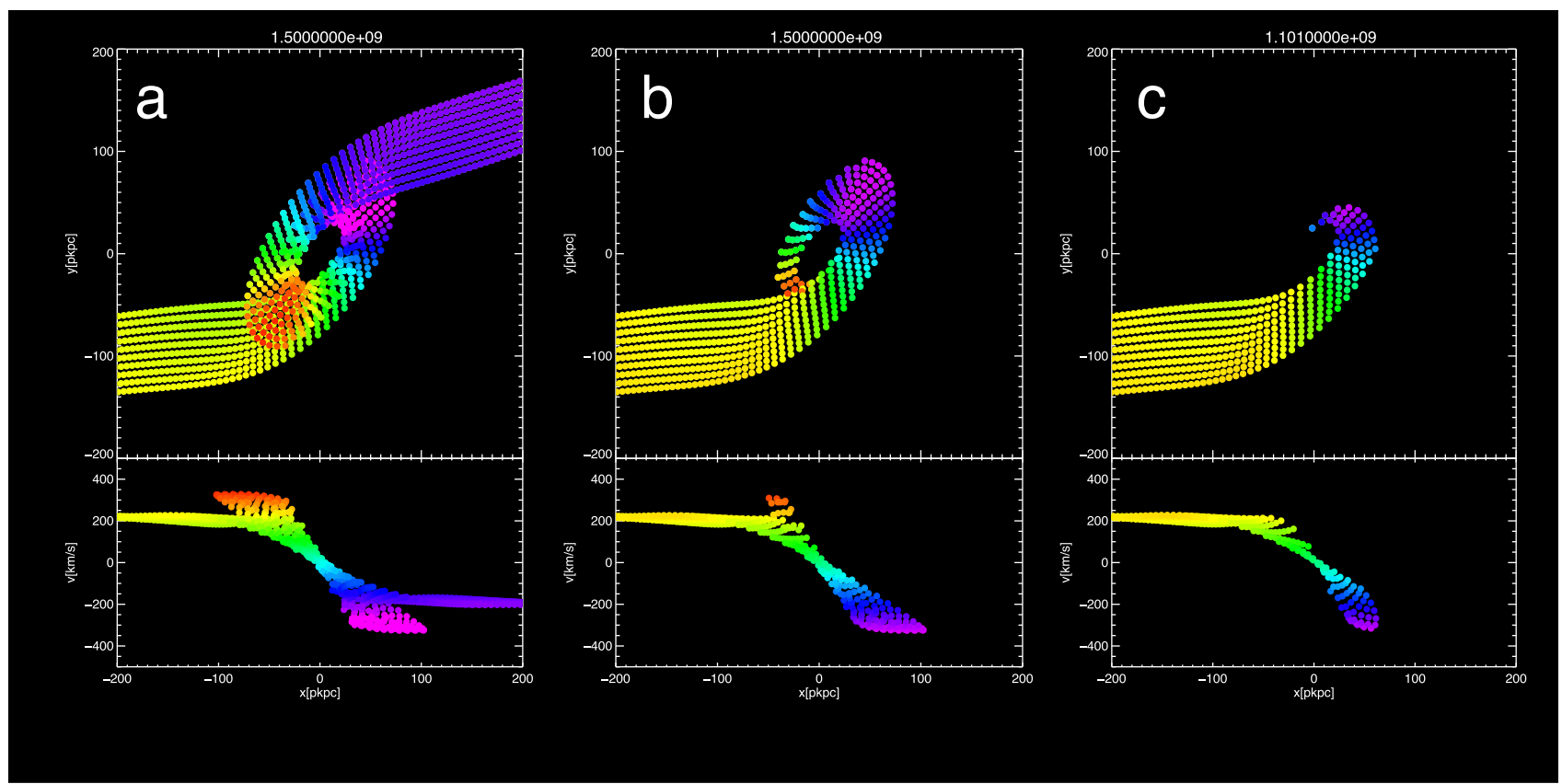

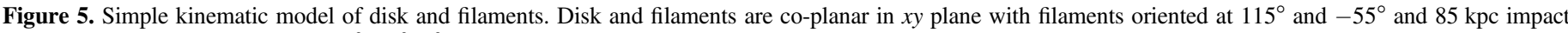

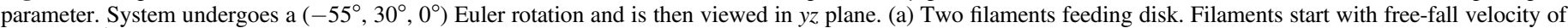

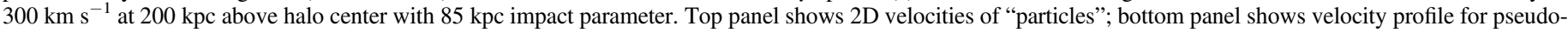
slit along filaments and disk. (b) Same as a with one filament, after 1.5 Gyr. (c) Same as a with one filament, after 1.1 Gyr.

note that there are two sources near the center (Figures 1 and 2, sources D and F) for which redshifts do not yet exist, and therefore star formation cannot be ruled out at or near the center.

\section{DISCUSSION}

We have presented evidence here that the HS1549+19 nebula is a second example of a cold-flow protogalactic disk, a galaxy in the process of forming through cold gas inflow from the cosmic web. The principle differences between the HS1549 +19 disk and that associated with UM287 are the even larger size of the new disk, the incomplete disk morphology, and the presence of multiple FCs. The discussion in M15 is relevant to this new system. In particular, radiative transfer effects can be ignored in this QSO-illuminated system as in the UM287 case, since the disk kinematics should be imprinted on the fluoresced Ly $\alpha$ kinematics and the double-peaked profile is too narrow to be resolved because of the low HI column density. Alternative explanations such as tidal interactions again do not provide a good model since the disk and filaments have a similar geometry and kinematic behavior. From Figure 1 it is evident that the disk is not a spurious product of QSO subtraction. There are numerous compact continuum sources in the system, and a few bright Ly $\alpha$ emitters (one associated with the southeastern portion of the disk). Those at the system redshift may be dwarf galaxies formed in the filaments prior to infall or within the forming disk. We showed in M14a that these objects are not spurious sources of the extended emission. However, there is no extended rest UV continuum component associated with the disk, suggesting that the majority of the gas has not yet reached the metallicity/column density threshold required to trigger significant star formation (M15), and the fluorescent brightness also suggests low Ly $\alpha$ absorbing dust content. The low gas metallicity suggests inflow from the cosmic web.
The orbital time of material in the outer disk is long, with $\tau_{\text {orb }} \sim 1.9\left(\frac{R_{\max }}{110 \mathrm{pkpc}}\right)\left(\frac{350 \mathrm{~km} \mathrm{~s}^{-1}}{v_{v}}\right) \mathrm{Gyr}, 0.85$ times the age of the universe and 0.97 times the virial timescale for a halo of this mass. Thus, the outer parts of the structure have had little time to equilibrate. The UM287 disk (M15) has an orbital time 0.3 times the Hubble time and 0.3 times the virial time. We might expect that the UM287 disk would be far more equilibrated than the QSO1549+19 protodisk. We observe that the protodisk is possibly incomplete, notably missing a sector of $\sim 120^{\circ}$ in the east, perhaps indicating an early stage of formation prior to disk equilibration. Perhaps the lack of emission in the "center" suggests that gas has not had time to spiral into the central disk. Alternatively, the eastern sector could be in the shadow of the QSO illumination cone. The large orbital time compared to the Hubble and virial time suggests that the disk must be only recently formed and therefore is demonstrably young and "protogalactic."

As in the case of UM287, the large diameter, high angular momentum, uniform gas surface density of the QSO1549+19 structure are like a "cold-flow protogalactic disk" or "cold spiral inflow" as predicted by simulations (Pichon et al. 2011; Stewart et al. 2011, 2013; Danovich et al. 2012, 2015). The morphology of this structure is reminiscent of the simulation presented in Figure 4 of Stewart et al. (2013). Such a protodisk will be fed by one or more filaments (Danovich et al. 2012, 2015; Cen 2014), and we presented evidence above that one or more filaments are present with consistent kinematics. The angular momentum parameter is even higher than that for UM287, and the disk is substantially larger even though the halo mass is lower, although still large.

The discovery of a second protodisk suggests that these objects may be common, and that cold-flow accretion is present at these redshifts and mass scales. A survey for other QSOilluminated cold-flow protogalactic disks will support a deeper 
understanding of where and how these systems form, and detailed study of these objects will offer major new insights into the complex process of galaxy formation.

This work was supported by the National Science Foundation and the California Institute of Technology. R.F.T. receives financial support from the UC Berkeley Miller Institute for Basic Research in Science.

\section{REFERENCES}

Behroozi, P. S., Conroy, C., \& Wechsler, R. H. 2010, ApJ, 717, 379 Bertoldi, F. 1989, ApJ, 346, 735

Birnboim, Y., \& Dekel, A. 2003, MNRAS, 345, 349

Blumenthal, G. R., Faber, S. M., Primack, J. R., \& Rees, M. J. 1984, Natur, 311,517

Bullock, J. S., Dekel, A., Kolatt, T. S., et al. 2001, ApJ, 555, 240

Cen, R. 2014, ApJL, 789, L21

Ceverino, D., Dekel, A., \& Bournaud, F. 2010, MNRAS, 404, 2151

Codis, S., Pichon, C., Devriendt, J., et al. 2013, MNRAS, 427, 3320

Danovich, M., Dekel, A., Hahn, O., Ceverino, D., \& Primack, J. 2015, MNRAS, 449, 2087

Danovich, M., Dekel, A., Hahn, O., \& Teyssier, R. 2012, ApJ, 422, 1732
Dekel, A., \& Birnboim, Y. 2006, MNRAS, 368, 2

Dekel, A., Birnboim, Y., Engel, G., et al. 2009, Natur, 457, 451

Ferland, G. J., Porter, R. L., van Hoof, P. A. M., et al. 2013, RMxAA, 49, 137

Goerdt, T., Dekel, A., Sternberg, A., et al. 2010, MNRAS, 407, 613

Hennawi, J. F., \& Prochaska, J. X. 2013, ApJ, 766, 58

Kereš, D., Katz, N., Weinberg, D. H., \& Davé, R. 2005, MNRAS, 363, 2

Martin, D. C., Chang, D., Matuszewski, M., et al. 2014a, ApJ, 786, 106

Martin, D. C., Chang, D., Matuszewski, M., et al. 2014b, ApJ, 786, 107

Martin, D. C., Matuszewski, M., Morrissey, P., et al. 2015, Natur, 524, 192

Matuszewski, M., Chang, D., Crabill, R. M., et al. 2010, Proc. SPIE, 7735, 77350P

Neufeld, D. A. 1990, ApJ, 350, 216

Pichon, C., Pogosyan, D., Kimm, T., et al. 2011, MNRAS, 418, 2493

Powell, L. C., Bournaud, F., Chapon, D., et al. 2011, in IAU Symp. 277, Tracing the Ancestry of Galaxies (Cambridge: Cambridge Univ. Press), 234

Prescott, M. K. M., Dey, A., Brodwin, M., et al. 2012, ApJ, 752, 86

Prescott, M. K. M., Martin, C. L., \& Dey, A. 2015, ApJ, 799, 62

Steidel, C. C., Giavalisco, M., Pettini, M., Dickinson, M., \& Adelberger, K. L. 1996, ApJL, 462, L17

Stewart, K., Kaufmann, T., Bullock, J., et al. 2011, ApJ, 738, 39

Stewart, K. R., Brooks, A. M., Bullock, J. S., et al. 2013, ApJ, 769, 74

Tillson, H., Devriendt, J., Slyz, A., Miller, L., \& Pichon, C. 2012, MNRAS, submitted (arXiv:1211.3124)

Trainor, R. F., \& Steidel, C. C. 2012, ApJ, 752, 39 